\title{
INEGALITES SOCIALES ET SCOLAIRES DANS L'ENSEIGNEMENT AGRICOLE
}

\author{
SOCIAL AND SCHOOL INEQUALITIES ON AGRICULTURAL EDUCATION \\ DESIGUALDADES SOCIAIS E ESCOLARES NO ENSINO AGRÍCOLA \\ DESIGUALDADES EN EL ÁMBITO SOCIAL Y ESCOLAR EN LA ENSEÑANZA RURAL
}

Laure Minassian*

Doutora em Ciências da Educação - Université de Paris VIII | França E-mail: laure-minassian@hotmail.fr

\begin{abstract}
REVISTA PEDAGÓGICA
Revista do Programa de Pós-graduação em Educação da Unochapecó | ISSN 1984-1566 Universidade Comunitária da Região de Chapecó | Chapecó-SC, Brasil Como referenciar este artigo: MINASSIAN, Laure. Inegalites sociales et scolaires dans l'enseignement agricole. Revista Pedagógica, Chapecó, v.16, n.32, p. 33-48, jan./jul. 2014.
\end{abstract}

RESUME: Les formations par alternance sont souvent invoquées pour faire réussir des élèves en échec dans les dispositifs plus classiques. Mais leurs effets sont-ils ceux escomptés ? Deux écoles qui relèvent de deux systèmes pédagogiques d'alternance dans l'enseignement agricole sont comparés. L'analyse comparative est centrée sur les productions écrites de ces derniers en début et en fin de formation. Les résultats de l'enquête remettent en cause la croyance en l'efficacité de certains dispositifs relevant de la pédagogie par alternance.

MOTS-CLES: Inegalites sociales et scolaires. L'enseignement agricole. Pédagogie par alternance.

\begin{abstract}
Courses are often invoked to lead with students who failed in conventional devices. Their effects are those expected? Two schools that are part of two educational systems of alternating in agricultural education are compared. The comparative analysis is centered on the productions written of these systems at the beginning and in the end of training. The results of the research deals with the belief in the efficacy of certain devices of pedagogy of alternation.
\end{abstract}

KEYWORDS: Social and school inequalities. Agricultural Education. Pedagogy of alternation

RESUMO: Cursos em alternância são frequentemente invocados para levar ao êxito escolar estudantes fracassados em dispositivos mais convencionais. Mas seus efeitos são aqueles esperados? Duas escolas que fazem parte de dois sistemas educacionais de alternância na educação agrícola são comparadas. A análise comparativa é centrada sobre as produções escritas dos referidos sistemas no início e no final da formação. Os resultados da pesquisa problematizam a crença na eficácia de determinados dispositivos da pedagogia de alternância.

PALAVRAS-CHAVE: Desigualdades sociais e escolares. Ensino Agrícola. Pedagogia da alternância. 
* Laboratoire: CIRCEFT-EScol, Université de Paris VIII
La baisse frappante des effectifs traditionnels de la formation agricole a conduit à ouvrir cet enseignement à un nouveau public constitué d'enfants d'employés et d'ouvriers en difficultés scolaires. Aujourd'hui, les enfants d'agriculteurs composent moins de 14\% de l'effectif tandis qu'ils étaient $80 \%$ dans les années 1970 (DGER, 2010 ; Grignon, 1975). À l'inverse la part des enfants d'ouvriers et d'employés hors secteur agricole a augmenté, elle représente presque $45 \%$ de l'effectif actuel (DGER, 2010) alors qu'elle n'atteignait qu'à peine $10 \%$ dans les années 1970 (Bonniel, 1972). Ces modifications ont en partie engendré une spécialisation de certaines institutions scolaires dans l'accueil de ces nouveaux publics quand d'autres se sont centrées sur le recrutement des derniers effectifs traditionnels. Les Maisons Familiales Rurales (MFR), écoles structurées en associations, accueillent massivement ces nouveaux élèves, alors que d'autres types d'institutions prestigieuses comme le lycée retenu dans le cadre de cette étude (dépendant des Frères de l'instruction Chrétienne), réputé pour son élitisme, tend à recruter dans les familles d'agriculteurs des enfants sans difficultés majeures à l'école. Si, de façon générale, la réussite scolaire dépend pour partie de l'origine sociale des élèves comme le montre entre autres le rapport Coleman (1966), confirmé par les travaux de Forquin (1982) et plus récemment par les enquêtes de la DEPP (2012), la proposition des MFR consiste à atténuer les inégalités par un enseignement qui, basé " sur la voie de l'alternance, réconcilie le jeune avec l'école grâce à une "pédagogie de projet" » (extrait document de la fédération MFR). Cette pédagogie contribue-t-elle pour autant à une transformation d'un premier parcours difficile vers celui d'une réussite scolaire? Si les MFR se distinguent par un discours institutionnel contre la fabrication de l'échec scolaire leur fréquentation provoque-t-elle une mutation dans le modèle de la reproduction (Bourdieu, Passeron, 1970)? En 1975, Claude Grignon, montrait que l'enseignement agricole était stratifié et concluait que les MFR enseignaient principalement des savoirs rudimentaires « abrégés", proches "des savoirs paysans traditionnels pour le rendre accessible et pour convaincre de son utilité » et qu'à l'inverse des savoirs "généraux, dépourvus de la base locale » étaient enseignés dans les lycées. Cette structuration était interprétée par l'auteur comme "l'équivalent de la division et de la hiérarchisation sociales du travail », participant de "l'ordre des choses » (Grignon, 1971), et ainsi de la reproduction. Les principaux résultats de son travail sont-ils toujours d'actualité?

Pour tenter de donner quelques pistes de réponses à ces questions, l'étude proposée s'appuie sur une analyse longitudinale de deux ans sur deux groupes d'élèves. L'angle d'étude est centré sur leurs productions écrites. Il s'agit plus précisément de rendre compte de l'évolution des pratiques de l'écrit de chacun des groupes au début de la 
formation et deux ans après. L'accent est porté sur les types d'objets valorisés par les élèves, en particulier lorsqu'ils rédigent des textes sur le thème du travail. La manière dont ils mettent en relation les objets qu'ils convoquent, la distance ou l'adhérence qu'ils expriment lorsqu'ils parlent avec ces objets sont des manières de faire langagières socialement situées qui permettent de mettre au jour des contrastes. Ces éléments servent à construire une analyse soucieuse de tenir compte de la multiplicité des dimensions qui se combinent entre principes pédagogiques et leurs effets, bien qu'ils ne réduisent pas complétement l'un à l'autre. Reste que cette relation entre principes et effets est ici particulièrement vive parce que les MFR prétendent constituer une voie alternative par le renversement des valeurs classiques de l'école, en proposant un apprentissage à partir du concret; tandis que l'école plus élitiste (le lycée des Frères) propose un enseignement en relation plus serrée avec les savoirs de la science agricole. Si la comparaison entre les groupes à différentes scansions du parcours de formation offre un certain point de vue descriptif, il nous faut cependant dépasser le simple constat qu'un type de pédagogie ne fait que reproduire, amplifier ou atténuer des inégalités de réussite initiales. Les principes éthiques et valeurs présents dans les discours que tiennent les praticiens en entretien constituent des indicesde compréhension des processusqui contribuent au renforcement d'écarts initiaux.

\section{CONTEXTE DE L'ETUDE ET CADRE THEORIQUE}

Unification de l'école: un prétexte pour reconduire l'enseignement spécialisé?

Les MFR sont une institution spécifique dans le paysage éducatif. Construites par des particuliers prêtres et laïcs dans les années 1930, elles répondent au départ à un besoin spécifique des familles contraintes de prolonger la scolarité de leurs enfants à la suite de la loi Jean Zay portant l'instruction obligatoire de 13 à 14 ans (1936) puis de 14 à 16 ans avec la réforme Berthoin (1959). Écoles de compromis, elles ont adopté un rythme permettant aux enfants de continuer à aider leurs parents sur l'exploitation familiale au prix de quelques semaines d'enseignement (7 semaines par an dans les années 1940) dans un lieu aménagé (une maison, un presbytère). L'unification de l'enseignement agricole (plus tardif que dans l'enseignement général) s'est achevée avec la reconnaissance de ces écoles dans le milieu des années 1980. Les MFR sont aujourd'hui habilitées à présenter leurs élèves aux diplômes nationaux, dépendent des mêmes curricula que les autres écoles d'enseignement agricole et le temps d'alternance est encadré par l'article L813-9 du Code Rural (il représente au maximum 50 \% du face-à-face pédagogique). Toutefois, cette reconnaissance n'a pas modifié en profondeur le statut particulier que les MFR s'attribuent et que les autres institutions de formation 
de ce segment lui attribuent. En effet, alors que les MFR revendiquent la paternité de l'alternance, «l'originalité de la formule » (pour reprendre une expression typique en MFR), - l'originalité - étant dans ce cas un terme valorisé, elles sont considérées par les personnels enseignants des autres institutions concurrentes comme une "école originale », au sens péjoratif d'une école " pas comme les autres».

Il faut noter d'ailleurs que la reconnaissance législative des MFR s'inscrit à un moment charnière dans l'histoire de l'enseignement agricole. En effet, dans le milieu des années 1980, les effectifs de cetenseignement s'effondrent (Lelorrain, 1999), en 20 ans près de la moitié des exploitations ont disparu du territoire français (Référentiel professionnel CGEA, 2008), coïncidant en outre avec le second mouvement de massification scolaire impulsé par Chevènement avec l'objectif d'atteindre " 80 \% d'une classe d'âge au baccalauréat d'ici à l'an 2000 » et donnant lieu à la création des baccalauréats professionnels. La conjoncture de ces deux événements a favorisé le ralliement des publics traditionnels vers les établissements prestigieux, laissant des places vacantes dans les établissements les plus populaires pour les nouveaux élèves de deux types: d'abord ceux originaires de l'agriculture, mais qui quelques années avant seraient sortis du système éducatif et sont contraints de poursuivre leur scolarité par incitation financière (les candidats à l'installation nés après 1971 bénéficient de dotations, de prêts bonifiés pour l'installation, sous couvert de l'obtention du baccalauréat, ils constituent une variante d' "enfants de la démocratisation scolaire ", Beaud, 2002), ensuite des élèves qui ne sont pas d'origine agricole, en difficultés dans les filières générales, et qui cherchent ou à qui est proposé une solution de recours que constituerait l'alternance. Autrement dit, si au départ, les MFR se sont adressées au public le plus populaire de la paysannerie, les familles qui ne pouvaient se passer de la main-d'œuvre enfantine, elles restent aujourd'hui une institution qui accueille les élèves dans les fractions les plus populaires du public qui compose l'enseignement agricole. On peut d'ailleurs se demander dans quelle mesure leur pleine intégration à l'enseignement agricole n'a pas eu vocation de créer des instituts spécialisés, avec l'objectif voilé que l'école «garde en son sein ceux qu'elle exclut » (Bourdieu, Champagne, 1992).

Pour comprendre ce que les institutions font aux individus, la seconde partie de l'enquête s'est déroulée dans un lycée prestigieux. Construit dans la deuxième partie du XVIIIe siècle sous le nom d'École Secondaire d'Agriculture (ESA), il a été un établissement d'élite à l'heure où l'accès au secondaire était réservé aux notables (Prost, 1986; Jardin, Tudesq, 1973). Accueillant les élèves à l'âge de la scolarité post-obligatoire, cette école est historiquement le lieu de la transmission de la science agricole. Elle a eu pour vo- 
cation de former les futurs chefs de domaines ainsi que les cadres de la paysannerie. Moins élitiste qu'hier, elle continue toutefois à accueillir un public héritier à plusieurs niveaux: héritiers tout d'abord parce qu'il s'agit d'élèves qui adoptent une culture double, à la fois scolaire, à la fois du travail et aux modes de pensées qu'il requière de par leur socialisation familiale ; héritiers encore puisqu'ils possèdent, au-delà de la culture de l'école et du travail, la possibilité de reprendre le capital d'exploitation de leurs parents (Minassian, 2013).

Dans les classes observées, les parcours des élèves de MFR sont non linéaires, ponctués de redoublements, d'orientations précoces (parfois dès la cinquième). Ces élèves ont pour certains d'entre eux connu des disqualifications symboliques (Millet, Thin, 2004) au collège, voire en primaire ou en maternelle. Leurs parcours sont très contrastés comparativement à celui plus ordinaire des élèves du lycée des Frères, ces derniers ayant peu redoublé, ont, dans leur grande majorité, suivi une troisième générale et obtenu le diplôme National du Brevet (sauf un). Ces contrastes sont aussi corrélatifs d'une distribution sociale car si la catégorie des enfants d'employés et d'ouvriers est dominante, elle est massivement représentée en MFR et très peu présente au lycée. Il en va ainsi de deux classes plus finement observées sur deux années (de la classe de bac pro première à la classe bac pro terminale). Dans la classe de MFR seuls trois élèves sont fils d'agriculteurs (le reste de la classe étant composée majoritairement d'enfants d'ouvriers ou d'employés non agricoles) alors qu'ils le sont tous dans celle du lycée des Frères. Les différences entre les groupes s'exercent en outre dans un rapport à l'école positif ou négatif. Les élèves qui, en difficulté, souhaitent s'extraire de l'école choisissent l'institution qui propose le plus d'alternance tandis que ceux qui hésitent davantage dans leur orientation - dont les notes auraient pu leur permettre de continuer dans l'enseignement général - choisissent l'établissement qui en propose le moins.

\section{L'écrit: une pratique sociale}

La comparaison des écrits des élèves est l'entrée retenue pour mieux comprendre des mécanismes de reproduction. Chacune des institutions étudiées forme un contexte soit propice à un apprentissage directement scolaire par un enseignement où les formes écrites sont enseignées avec une grande systématicité et de façon décontextualisée, soit propice à la valorisation de la vie quotidienne par la description de pratiques de travail empiriquement constatables comme préalable à l'entrée dans un apprentissage académique.Nous faisons l'hypothèse avec Budach et Patrick (2012), Barton et Hamilton, (2010) que les pratiques de l'écrit sont façonnées par l'environnement immédiat dans lequel elles sont inscrites ainsi que par les mouvements et trajectoires des individus. Les éléments langagiers sur les- 
quels reposent nos analyses de pratiques littératiées, visent à rendre compte de la manière dont les élèves mettent en relations les objets qu'ils mobilisent, des liens qu'ils construisent entre ces objets comme indices de création de sens. Néanmoins comme dans le langage oral, l'écrit ne peut être pensé comme le reflet de productions construites préalablement par le sujet et qu'il suffirait de retrouver au moyen d'une méthodologie efficace, parce qu'à rebours l'écrit travaille les manières de penser, de catégoriser le monde (Goody, Watt,1963). C'est donc un ensemble d'indices relevés à partir des écrits qui ici interrogé au sens d'une littératie, conçue comme activité socio-cognitive et socio-culturelle (Street, 1993).

Travailler sur les écrits des élèves ne relève toutefois pas toujours du registre textuel, il existe un ensemble d'éléments sémiotiques tels que la mise sous forme de tableau, la liste par point, l'emploi de systèmes mathématiques, de plans, etc. qui contribuent du sens que les élèves construisent quand ils écrivent. L'écrit est donc pensé à partir d'un ensemble de modes de faire scripturaux.

\section{METHODOLOGIE}

Les enquêtes de terrain ont été réalisées pendant les deux dernières années de formation conduisant au baccalauréat agricole pour chacun des groupes. Le choix d'analyser ce que font les élèves à ce niveau de diplôme tient à sa spécificité symbolique à au moins deux niveaux. Tout d'abord il constitue un sésame depuis qu'il est devenu,dans les années 1980, une des conditions pour obtenir des aides financières pour l'installation d'une exploitation agricole. Cette incitation participe d'une démocratisation dans l'accès au niveau IV et a eu pour effet de permettre à des élèves de poursuivre leur scolarité là où ils se seraient arrêtés auparavant. Il est en outre un diplôme mis en œuvre dans une politique plus globale qui accompagne le second mouvement de massification contribuant, de façon cumulative au premier effet, à l'intégration de nouveaux élèves. Ces deux effets solidaires de la création du diplôme font de ce niveau de formation un lieu pertinent à interroger au regard de la réussite ou de l'échec des élèves et de façon plus spécifique de la reproduction des inégalités sociales et scolaires dans l'école.

Le module professionnel qui a fait l'objet d'observations plus systématiques a été choisi relativement à la position qu'il occupe dans le curriculum à deux points de vue puisque tout d'abord il est le module à l'interface entre l'école hors et dans les murs, c'est-à-dire celui qui est le plus en relation avec le stage permettant idéalement la mise en œuvre d'une pédagogie ouverte sur le monde du travail dont se réclament les MFR. Il est ensuite celui qui possède le plus fort coefficient pour l'obtention du baccalauréat requérant ainsi un fort investissement de leur part. Les élèves sont attendus à composer des dossiers théma- 
tiques sur les pratiques d'élevage. La noteattribuée à ce travail se scinde en deux parties : une certificative (sous la forme d'un Certificat en Cours de Formation), l'autre terminale lors d'un oral face à un jury d'examen.Les dossiers qui composent notre corpus sontdonc, de ce point de vue, des copies d'épreuves en vue de l'obtention du diplôme. Plus de 3000 feuilles (300 dossiers) ont fait l'objet du recueil. Les 90 dossiers analysés dans le cadre de cette contribution ont été rédigés à trois moments de la formation (alimentation des vaches laitières, la qualité du lait, les animaux de renouvellement). Ces dossiers, parce que produits à différents moments du parcours ont été choisis pour rendre compte d'évolutions longitudinales.

Dans un second temps, les pistes de lecture proposées pour comprendre les évolutions constatées concerneront davantage les MFR puisque les littératies mises en œuvre par les élèves de cette institution interrogent davantage que celles du lycée des Frères. Il s'agira alors de comprendre en quoi les valeurs de l'institution peuvent constituer un obstacle à la construction d'écrits académiques. Plus précisément il s'agira de mettre au jour la manière dont se manifestent, dans les discours des enseignants en entretien, ces principes pédagogiques et valeurs autour de l'école ouverte sur le monde. Cinq entretiens semi-directifs longs permettent de poser quelques jalons explicatifs au sujet des productions des élèves.

\section{RESULTATS}

\section{Pluralité des littératies scolaires}

Les objets valorisés par les élèves sont-ils essentiellement empiriques? La manière de présenter ces objets s'apparente-t-elle à une chronologie telle qu'elle se réalise dans la vie quotidienne ou les élèves en construisent-ils des catégories plus abstraites?

Les dossiers analysés ont fait l'objet d'une étude systématique sur chacun des segments signifiants, il peut s'agir d'un groupe de mots, d'une phrase, de plusieurs phrases par exemple. La présence ou l'absence de relation entre les objets mobilisés par les élèves est une première catégorie retenue pour rendre compte de l'évolution de leurs écrits. Si dans la suite de ce texte ces types de relation feront l'objet de développements plus approfondisen focalisant l'analyse sur le registre mobilisé (registre descriptif, narratif, chronologique, affectif, scientifique, etc.), c'est d'abord la juxtaposition ou l'atomisation des objets choisis qui fait l'objet du codage. Chaque segment de signification est donc analysé au regard des segments qui le précèdent et le succèdent et avec l'ensemble du texte produit. Typiquement un texte constitué par une suite de segments posés les uns à la suite des autres est codé sous la catégorie des actes langagiers atomisés. L'extrait ci-dessous est donné à titre illustratif.Les segments signifiants sont posés successivement sans relation entre eux, ce quid'ailleurs empêche 
1 L'élève utilise deux fois le terme «quantité », l'un des deux termes est possiblement à remplacer par le terme «qualité ». l'élève de répondre à la consigne qui consistait à dégager des objectifs d'un éleveur:

Les objectifs de l'éleveur.

- L'exploitant utilise et valorise au printemps ses fourrages et le pâturage.

- L'utilisation des fourrages stockés se fait par quantité et non par quantité ${ }^{1}$.

- Les concentrés interviennent en plus des fourrages pour l'équilibre azote/énergie et la production laitière. (Sébastien)

Un second exemple permet de caractériser ce qui est analysé en tant que texte constitué de segments mis en cohérence. Dans ce second extrait, l'élève pose un descriptif de l'exploitation et des pratiques d'élevage pour, dans un second temps, les interroger:

Sur cette exploitation la SAU (Surface Agricole Utile) représente 125 ha (hectares) principalement constituée d'herbe car la surface en herbe représente $60 \%$ de la SAU et $72 \%$ de la SFP (Surface fourragère Principale). Mais comparativement à cela la surface pour le pâturage est de 25 ha pour le cheptel laitier soit $24 \%$ de la SFP. Pourquoi un tel choix? (Jordan)

D'un point de vue plus global, les segments signifiants des élèves, leur atomisation versus juxtaposition ont fait l'objet d'une quantification (cf. tableau ci-dessous). La comparaison porte sur deux séries (plus de 1000 segments) et permet de rendre compte d'évolutions entre le début et la fin de la formation.

Tableau 1. Catégorisation à partie de 1.241 segments de signification

\begin{tabular}{|l|c|c|c|c|c|c|}
\hline Institutions & & \multicolumn{1}{|c|}{ MFR } & & & \multicolumn{1}{|c|}{ Lycée } & \\
\hline $\begin{array}{l}\text { Catégorisation } \\
\text { des segments } \\
\text { de signification } \\
\text { dans les } \\
\text { dossiers des } \\
\text { élèves }\end{array}$ & $\begin{array}{l}\text { Segments } \\
\text { de } \\
\text { signification } \\
\text { atomisés }\end{array}$ & $\begin{array}{l}\text { Segments de } \\
\text { signification } \\
\text { juxtaposés }\end{array}$ & Total & $\begin{array}{l}\text { Segments de } \\
\text { signification } \\
\text { atomisés }\end{array}$ & $\begin{array}{l}\text { Segments } \\
\text { de } \\
\text { signification } \\
\text { juxtaposés }\end{array}$ & Total \\
\hline $\begin{array}{l}\text { Dossier en } \\
\text { début de } \\
\text { formation }\end{array}$ & 290 & 135 & 425 & 199 & 138 & 337 \\
\hline $\begin{array}{l}\text { Dossier en fin } \\
\text { de formation }\end{array}$ & 217 & 47 & 264 & 83 & 132 & 215 \\
\hline
\end{tabular}

Letableau révèle des traits communs et des différences entre les groupes. Tout d'abord, on observe que dans la durée, les élèves tendent à réduire quantitativement leurs écrits et ce quel que soit le groupe observé. Quantitativement encore les élèves de MFR composent des dossiers plus longs que leurs homologues du lycée au début comme à la fin de la formation. En revanche qualitativement des différences fortes apparaissent. Alors qu'au début de la formation les élèves de MFR produisaient autant de segments juxtaposés que les élèves du lycée, la production de juxtapositions baisse significativement entre le premier et le dernier 
2 Nous reproduisons les textes des élèves fautes syntaxiques comprises. dossier composé pour les élèves de MFR. À l'inverse le nombre de segments juxtaposés augmente de façon très sensible pour les élèves du lycée. La comparaison entre les segments atomisés et les segments mis en cohérence font apparaître des variations inversement proportionnelles entre les groupes. En effet, les éléments langagiers épars, sans mise en lien, baissent approximativement de $60 \%$ pour les élèves du lycée, alors que pour les élèves de MFR ce sont les éléments mis en relation qui connaissent une chute très forte, d'un peu plus de $65 \%$.

Ces éléments, comparés entre début et fin de formation, interrogent les parcours mêmes des élèves et leurs orientations.Car bien qu'en MFR la trajectoire scolaire des élèves soit balisée par un ensemble de difficultés, les performances des deux groupes ne sont pas si éloignées au début de la formation. Cependant, les écarts deviennent très importants au fur et à mesure des deux années. Les différences qualitatives apparaissent en de nombreux endroits, et alors qu'au début de la formation les élèves de MFR tentent de mettre en avant les intérêts et les limites d'une pratique d'élevage par exemple, c'est-à-dire d'en construire des indices de sens, ils rabattent à mi-parcours de la formation leurs écrits sur la narration d'objets observables, rédigent des descriptifs que pourrait faire un visiteur quelconque sur une exploitation. Ces effets sont encore plus manifestes dans la mesure où en fin de formation les élèves ne rédigent plus que des réponses les plus brèves possibles aux questions d'exercices ou de cours. Le cas du dossier à mi-parcours est charnière de ces deux postures. Pour décrire des pratiques d'éleveurs relatives aux cycles de reproduction des animaux, les élèves de MFR écrivent ce qu'ils voient empiriquement, parfois rapportent les propos du maître de stage. Nous en donnons ci-dessous quelques extraits:

Tout $^{2}$ les soirs l'agriculteurs vient voir ses vaches (vers 22h) pour repérer les vaches en chaleur. Si une vache n'est pas en chaleur au bout de 50 jours, elle est contrôlée par l'inséminateur et le vétérinaire (Ewen).

Repérage des chaleurs: ils sont tous repérés grâce à la vue, et si les vaches sont en chaleur, on regarde sur le bulletin du contrôleur laitier si elles sont bonnes à inséminer.(Julien)

l'éleveur à sa maison à côté, se qui permet de surveiller aisément, les mises bas(Jérémie)

Les vêlages ont le plus souvent lieu au pré, avec une observation régulière. Les vêlages se font sans aide, lorsque tout se présente bien. L'éleveur préfère laisser faire la nature. (Jérôme)

La surveillance des chaleurs est également réalisée le matin, le midi et le soir, en restant avec les animaux environ 15 minutes (Valentin).

À l'inverse, les élèves du lycée s'appuient sur des processus, des savoirs pour expliciter des pratiques d'élevage 
liées à ces cycles. Les formes de mise en relation témoignent de manières de penser qui se développent dans une direction presque opposée à celle des élèves de MFR. Les élèves du lycée soulignent des contrastes entre deséléments constatés et en construisent une problématique, cette tendance est beaucoup moins fréquente pour les élèves de MFR (excepté pour deux d'entre eux):

Tout d'abord l'intervalle vêlage 1ère IA (première Insémination Artificielle) paraît réparti à près de $50 \%$ sur les données supérieur à 90 jours (après le vêlage) on pourrait se poser la question y a-t-il un problème de mise à la reproduction (mauvaise détection de chaleurs, déficit énergétique ou problème sanitaire). En fin de compte le pourquoi du comment reste facile à expliquer l'éleveur veut mettre ses animaux à la reproduction lorsqu'ils se sont « refaîtes » pour avoir plus de chance de réussite.

L'explication donner au-dessus se confirme car le taux de réussite en 1ère en IA ma paraît très convenable car on a plus de $56 \%$ et plus de $25 \%$ en 2ème IA donc une bonne fertilité du troupeau laitier (Jordan).

Le texte reproduit ci-dessus, au-delà des maladresses de l'élève, atteste d'un pluri-référencement prenant sa source dans des indicateurs choisis par l'élève (le nombre d'IA et la période à laquelle elles sont pratiquées), de pourcentages pour rendre compte d'une cohérence entre des éléments non habituels (de la mise en évidence d'un écart), de propos rapportés du maître de stage ( $"$ En fin de compte le pourquoi du comment reste facile à expliquer l'éleveur veut mettre ses animaux à la reproduction lorsquỉs se sont "refaîtes » pour avoir plus de chance de réussite »). En outre, l'élève traite les objets techniques sur un registre scolaire scientifique. Il met en circulation un ensemble de pratiques au regard de leur opérationnalité (le meilleur rendement possible que permettent ces pratiques) dans un schéma énonciatif propre à celui de la science appliquée : décrire des constats, formuler des hypothèses (mauvaise détection de chaleurs, déficit énergétique ou problème sanitaire), mettre à l'épreuve ses hypothèses, même si ces dernières ne sont pas toutes traitées.

Dans l'exemple qui suit, la mise en avant des savoirs de la science appliquée scolaire devient l'articulation par laquelle l'élève répond à la problématique qu'elle pose:

Le pourcentage d'infertilité a augmenté de $10 \%$ en 2008-2009, du à une chute de $32 \%$ des vaches fécondes en 1ère IA. L'intervalle vêlage-vêlage a augmenté de 11 jours en 2008-2009. L'année 2008-2009 est plutôt négative, au niveau des résultats ceuxci s'explique par les problèmes sanitaires rencontrés sur l'exploitation (...). Pour la réforme, il y a eu une augmentation de $9 \% \mathrm{du}$ 
$<?>\mathrm{Nb}$ : «9 $500 \mathrm{~kg}$ de lait/vache/an » signifie que le niveau de production est élevé et ce niveau a des conséquences sur le métabolisme animal. taux de réforme en 2008 du à des problèmes sanitaires (FCO et paratuberculose) qui ont touchés le troupeau laitier (Amélie).

Bien que le questionnement de l'élève n'apparaisse pas de façon aussi explicite que dans la production précédente, elle tente de poser des raisons qui expliquent les écarts qu'elle constate entre deux années. Cette centration sur les savoirs de la science appliquée scolaire est investie encore par cet autre élève:

La part la plus importante des réformes est l'infertilité. Cela peut ce comprendre a un niveau d'étable de $9500 \mathrm{~kg}$ de lait ${ }^{3}$. De plus, les boiteries on a une part importante dans le taux de réforme car il y a une présence de dermatite sur l'exploitation (Arnaud).

La mise en correspondance entre savoirs scientifiques (sanitaires: dermatite, FCO, paratuberculose, poids de l'animal comme cause d'infertilité), techniques et pratiques compose les raisonnements les plus fréquents au lycée. La pluralité des éléments pris en compte : statistiques, propos rapportés, inférences, hypothèses, savoirs mobilisés, mais aussi l'intégration de schémas et graphiques construits par les élèves tend à dessiner des écrits scientifiques scolaires, dans une polyphonie, participant d'une posture d'élève, parfois proche d'un responsable d'exploitation. Participe encore de cette analyse, la hiérarchisation des énoncés des élèves construits sur la description, permettant de mettre en œuvre un questionnement, de poser des hypothèses et de les tester avec des savoirs techniques ou scientifiques étayés par des éléments objectifs (statistiques, graphiques, etc.).

Ces quelques éléments de l'analyse questionnent la pertinence de la "pédagogie de la réussite » prônée en MFR puisque les apprentissages tels qu'ils s'actualisent dans les écrits des élèves se différencient au fur et à mesure de la formation. À mi-parcours les registres mobilisés sont narratif et chronologique pour les élèves de MFR, scientifique pour les élèves du lycée.Pourquoi les écarts se creusent-ils de façon sensible alors qu'au départ il semble n'y avoir qu'une différence de degré ? S'il existe de multiples causes expliquant potentiellement ces différences, y joue, nous semble-t-il, des idéologies vives qui circulent dans les principes pédagogiques de l'institution dont rendent compte les entretiens passés aux enseignants de MFR. Les contenus de ces discours vont faire l'objet des lignes suivantes.

\section{PARTIR DES BESOINS DES ELEVES : UNE PEDAGOGIE ALTERNATIVE OU UN PRET-A- PENSER?}

Les principes pédagogiques constituent-ils un des éléments qui participe des écarts constatés? Rappelons qu'historiquement les MFR ont été un compromis entre l'instruction obligatoire et le besoin de main-d'œuvre, 
qu'elles ont évolué en développant des discours, des valeurs centrés sur les besoins des élèvesprésentés en opposition potentielle à ceux de l'école. Proche d'un anti-intellectualisme, c'est moins la diffusion des savoirs scolaires que de partir de ce qui fait sens aux élèves, de ce qu'ils rencontrent dans leurs expériences qui permettraient des acquisitions plurielles. Cette inversion des valeurs a d'ailleurs été nourrie par des conceptions de l'éducation nouvelle, Henri Wallon ayant participé de la refondation des MFR. De ce point de vue l'alternance en MFR n'est pas un simple aller-retour entre l'école et l'entreprise, maisest conçue comme favorisant une entrée dans la théorie des questions. La reprise de ce qui étonne les élèves dans l'activité de travail, la mise en relation de leurs questionnements avec d'autres questions plus globales permettrait de tendre vers un processus d'acquisition scolaire, voire induiraient des rapports aux savoirs revisités par les élèves. Identifier des besoins, prendre en considération les intérêts d'autrui, sont des éléments qui s'inscrivent dans un idéal qui consiste à se donner d'autres mondes possibles. Cet idéal est porté par les enseignants comme en témoignent les modes de réappropriation dans leurs discours. Des entretiens longs (parfois de deux heures), auprès de cinq enseignants différents mettent au jour un fonctionnement pédagogique qui repose sur la conviction que l'alternance peut tout. Nous en donnons ici quelques extraits à titre illustratif:

Michel: c'est-à-dire qu'ici on partage un peu la même conviction/et le principe/on observe un sujet ou un thème/concernant la conduite d'une production animale ou végétale/à partir d'un questionnaire élaboré à partir du questionnement des élèves avant le stage/qu'ils testent en stage/et en revenant à l'établissement on fait une mise en commun plus ou moins détaillée de ce qui a été observé (Enseignant MFR depuis plus de 5 ans).

Murielle: Moi j'ai découvert l'alternance à l'ENSA d'Angers/hein/on était deux mois en cours/deux mois en stage/et vraiment je m'suis dit/c'est génial quoi/l'expérience sur les lieux de stage est irremplaçable et euh/ je m'suis dit/moi j'ai envie de former des/de travailler avec l'alternance ou l'apprentissage/à l'époque je ne savais pas trop/mais je trouvais que le système Maison Familiale était adapté pour euh/pour motiver les jeunes/pour trouver un emploi par la suite plus facilement (Enseignante MFR depuis plus de 20 ans).

Jean: [...] des notions liées à des événements historiques quel'on pourrait avoir un moment donné/par exemple de se dire au moment de l'alternance/ben de dire aux jeunes de rencontrer des anciens combattants/ des gens qui ont vécu la guerre d’Algérie/ enfin je n'en sais rien moi/ mais de se dire « tiens pourquoi ne pas/à partir du milieu 
professionnel/ne pas aborder/des questions/ des notions qui sont liées à des matières générales (Enseignant MFR depuis plus de 20 ans).

Daniel: [...] c'est un petit peu ce qui m'a motivé pour m'intéresser à ce système de formation par alternance/ d'avoir compris que dans une formation technique professionnelle/il y avait absolument la nécessité de se référer à/à euh/au milieu professionnel par des stages et par une insertion dans ce milieu euh par une insertion euh/une forte insertion/hein/donc c'est ce qui m'a amené à/à ce choix un petit peu de l'enseignement/dans ce euh/dans ce type de formation/et donc/là/j'ai découvert dans les Maisons Familiales et instituts des gens qui avaient aussi ces convictions/ (Enseignant MFR depuis plus de 35 ans)

Ces quelques extraits d'entretiens montrent que les personnels enseignants investissent dans leurs discours un idéal de l'alternance et de ce que représente à cet égard l'institution MFR. Tout se passe comme si les convictions liées à l'alternance, les valeurs de partage et de solidarité présentes dans les discours des enseignants semblent contribuer d'une idéologie en tant que «le propre de l'idéologie est d'imposer des évidences comme évidences. Le sujet fait corps avec les idées qui lui ont été " inconsciemment " transmises " (Bourdieu, Passeron, 1964). Dans cette perspective, la pédagogie des MFR serait hypothétiquement une mystification faisant obstacle à la reprise des principes pédagogiques à l'aune des effets de l'action pédagogique. D'ailleurs lors de la passation des entretiens, à la question " en quoi l'alternance peut constituer un obstacle » un enseignant répondra ne s'être «jamais posé la question ", trois désignerons le maître de stage comme ne jouant " pas le jeu » et un autre utilisera la parabole de la germination pour expliquer en quoi les graines (c'est-à-dire les savoirs) aujourd'hui semées croîtront plus tard, " parfois bien après la formation ». Jamais l'alternance et l'idéal qui l'accompagne ne seront remis en cause. Cette " mystagogie » (Lahire, 2000) où on ne sait quand les élèves recueillent les fruits de cette expérience à la fois scolaire et du travail, participe, sous couvert d'aider les élèves, de la reproduction et empêche de penser ce que les élèves font réellement avec l'écrit et en quoi les pratiques pédagogiques y contribuent.

\section{CONCLUSION. "UNE PEDAGOGIE DE PROJET": ALTERNATIVE A L'ECHEC SCOLAIRE?}

Ici comme ailleurs l'école serait une instance de reproduction d'une culture dominante en distribuant les élèves dans des établissements plus ou moins valorisés. Ceux issus des classes dominantes, formés dans l'ancienne école élitiste, seraient favorisés par la transmission d'une culture scolaire au profit de la conservation du patrimoine 
familial, voire de son amélioration alors que le public plus populaire, moins agricole, parfois urbain, le plus souvent profane serait relégué dans un établissement où la culture du travail au sens de faire est première. Dans cette perspective, l'enseignement agricole est une illustration du modèle théorique de Bourdieu et Passeron (1964, 1970).

Il faudrait bien sûr reconduire cette étude à plus grande échelle pour confirmer, infirmer ou nuancer le propos, les groupes étant ici très contrastés. Reste que comparer des littératies dans une perspective longitudinale permet de questionner les prétentions d'une certaine pédagogie de l'alternance. Alors même que les MFR sont décrites dans la littérature de l'institution comme des écoles progressistes favorisant la « réconciliation entre le jeune et l'école » (document MFR), celle-ci ne semble pas se réaliser dans les apprentissages des élèves tels qu'ils se manifestent dans leurs productions écrites. En effet, au fur et à mesure de la formation, les élèves de MFR s'éloignent des mises en relation académiques pour produire un ensemble de descriptions empiriques et chronologiques. À l'inverse, les élèves du lycée, confrontés à un environnement plus académique produisent des écrits de plus en plus complexes et pluri-référencées. Un des résultats de l'enquête est donc que les différents types d'institutions tolèrent différents usages de l'écrit et différenteslittératies.

Mais cette pluralité de littératies scolaires pose la question des arrangements évaluatifs (Merle, 2012). Car malgré que le diplôme soit commun ainsi que les critères d'évaluation, la notation donne lieu à un CCF (Certificat en Cours de Formation)c'est-à-dire une note que l'enseignant de référence attribue seul.Dans cette perspective, les critères d'évaluation peuvent largement faire l'objet de réinterprétations, de compromis et de transformations plus ou moins conscients (Bonniol, Vial, 1997). Puisque chaque évaluateur corrige dans le sein de son institutionpar rapport à des contraintes interpersonnelles et/ou institutionnelles il pourrait être énoncé de façon un peu radicale que cette organisation du diplôme contredit l'idée même d'un diplôme national. En outre, le processus d'interprétation et de prise de décision en cours de correction des productions des élèves, parce qu'il se situe à l'interne des institutions, ne permet pas davantage de remettre en cause des principes qui guident l'action pédagogique. Ainsi les principes pédagogiques des MFR qui s'actualisent sous la forme d'une conviction, perdurent et participent potentiellement et parmi d'autres causes, de la conservation des inégalités de départ, voire de leur accroissement.

\section{BIBLIOGRAPHIE}

BARTON, D.; HAMILTON, M. La littératie: une pratique sociale. Langage et société. Paris, Maison des sciences de l'homme, n.133, p. 45-62, 2010. 
BONNIEL, J. La réussite scolaire dans le cadre d'une institution privée de formation: le cas del'UNMFREO. Caluire: Economie et Humanisme, 1972.

BONNIOL, J-J; VIAL, M. Les modèles de l'évaluation (textes fondateurs et commentaires). Paris, Bruxelles: De Boeck, 1997.

BOURDIEU, P.; CHAMPAGNE, P. Les exclus de l'intérieur. Actes de la recherche en sciences sociales. Paris, Seuil, v. 91-92, mars, p. 71-75, 1992.

BOURDIEU, P. ; PASSERON, J-C. Les héritiers. Les étudiants et la culture. Paris: Editions de Minuit, 1964.

BOURDIEU, P.; PASSERON, J-C. La reproduction. Eléments pour une théorie du système d'enseignement. Paris: Editions de Minuit, 1970.

BEAUD, S. 80\% au bac... et après? Paris: La Découverte, 2002.

BUDACH, G.; PATRICK, D. «Chaque objet raconte une histoire» Les pratiques de littératie chez des Inuits en milieu urbain. Recherches en didactique des langues et des cultures: Les Cahiers de l'Acedle. Paris, Acdle, v. 9, n. 2, 2012.

COLEMAN J. S. ; CAMPBELL E. Q. ; HOBSON C. J. ; MCPARTLAND J.; MOOD A. M.; WEINFIELD F. D. ; YORK R. L. Equality of Educational Opportunity. Washington, DC: US Office of Education, 1996.

DGER 2010, Études statistiques. Disponible dans: http://www. chlorofil.fr/systeme-educatif-agricole/insertion-scolaire-sociale-et-professionnelle/etudes-articles-et-statistiques/insertion-scolaire-et-professionnelle-des-diplomes.html\#c7960 Consulté en : 10/02/2014.

DEPP (2012), Conditions de scolarisation et facteurs de réussite scolaire. Education et formations. (Quéré, M., dir), n. 82, dec.2012.

FORQUIN, J-C. La sociologie des inégalités d'éducation: principales orientations, principaux résultats depuis 1965. Revue Française de Pédagogie. École Normale Supérieure de Lyon, n. 48, p. 90-100, 1982.

GOODY, J.; WATT, I. P. The consequences of literacy. Comparative Studies in Society and History. Cambridge University Press, v. 5, p. 304-345, 1993.

GRIGNON, C. L'ordre des choses, les fonctions sociales de l'enseignement techniques. Paris: Minuit, coll. Le sens commun, 1971. 
GRIGNON, C. (1975), L'enseignement agricole et la domination symbolique de la paysannerie. In: Actes de la recherche en sciences sociales. Paris, Seuil, v.1, n.1, p.75-97, jan-vier, 1975.

JARDIN, A.; TUDESQ, A-J. La France des Notables. Paris: Editions du Seuil, 1973.

LAHIRE, B. Savoirs et techniques intellectuelles à l'école primaire. In: VAN ZANTEN, A. (org.). L'école. L'état des saviors. Paris: La Découverte, p. 170-178, 2000.

LELORRAIN, A-M. L'enseignement postscolaire agricole. In: CHARMASSON, T.; DUVIGNEAU, M.; LELORRAIN, A-M.; LE NAOU, H. (orgs.). L'enseignement agricole: 150 ans d'histoire, évolution historique et atlas contemporain. Dijon: Educagri editions, 1999.

MILLET, M.; THIN, D. La déscolarisation comme parcours de disqualification symbolique. In: GLASMAN D.; EUVRARD, F. (orgs.). La déscolarisation. Paris: La Dispute, 2004.

MERLE, P. La ségrégation scolaire. Paris: La Découverte, 2012.

MINASSIAN, L. Des formes scolaires différenciées : unification du système éducatif ou réseaux de scolarisation? L'exemple de l'enseignement professionnel agricole. Thèse. Paris VIII (sous la direction de Bautier, E.), 2013 .

PROST, A. L'enseignement s'est-il démocratisé? Paris: Presses Universitaires de France, 1986.

STREET, B. Cross-cultural Approaches to Literacy. Cambridge: Cambridge University Press, 1993. 